\section{PS1:16 THE PRESENCE OF AUTOANTIBODIES TO MULTIPLE KILLER CELL IMMUNOGLOBULIN-LIKE RECEPTORS IS ASSOCIATED WITH NEPHRITIS IN SLE PATIENTS}

N Hagberg, D Leonard, G Nordmark, L Rönnblom. Rheumatology and Science for Life Laboratories, Department of Medical Sciences, Uppsala University, Uppsala, Sweden

\subsection{6/lupus-2018-abstract.64}

Purpose Natural killer cell cytotoxicity is regulated by inhibitory receptors recognising HLA. These include the CD94/ NKG2A and the killer cell immunoglobulin-like receptors (KIR). Previously we described functional autoantibodies to CD94/NKG2A, in patients with systemic lupus erythematosus (SLE). Here we investigated whether patients with SLE, primary Sjögren's syndrome (pSS) or systemic sclerosis (SSc) have autoantibodies to KIR-receptors and whether presence of such autoantibodies correlates to clinical manifestations.

Method HEK293-transfectants expressing KIR2DL1, KIR2DL2, KIR2DL3, KIR3DL1, KIR3DL2, KIR2DL4, KIR2DS2 or KIR2DS4 were incubated with serum from 191 SLE, 121 pSS, 48 SSc patients and 100 healthy donors. Binding of human IgG to each transfectant was determined using flow-cytometry and the median fluorescence intensity (MFI) was divided by the MFI of untransfected cells. The cut-off for autoantibody-positivity was set at the mean +4 standard deviations of healthy donors. Clinical data were tested for association to the presence of anti-KIR autoantibodies using Fischer's exact test.

Results Autoantibodies to KIR-receptors were identified in $23.0 \%$ of SLE, $10.7 \%$ of pSS and $12.5 \%$ of SSc patients compared to $4.0 \%$ of healthy individuals. Anti-KIR antibodies to all eight receptors studied were detected in SLE and pSS sera, whereas SSc sera reacted with four of the receptors. The highest titers of anti-KIR antibodies were found in SLE sera. All KIR-positive healthy donor sera and the majority of KIR-positive pSS (76.9\%) and SSc $(50.0 \%)$ sera reacted with 1 KIR-receptor. In contrast, $36.3 \%$ and $22.6 \%$ of the anti-KIR-positive SLE sera reacted with $2-3$ and $>3$ KIR-receptors, respectively. Autoantibodies to $>3 \mathrm{KIRs}$ were associated with an increased risk for lupus nephritis (80.0\% vs $27.2 \%$, $\mathrm{p}=0.001)$, an increased number of ACR criteria fulfilled (7 vs 6 , $\mathrm{p}=0.02)$, presence of anti-Sm $(50.0 \%$ vs $13.6 \%, \mathrm{p}=0.01)$ and anti-RNP $(70.0 \%$ vs $23.1 \%, \mathrm{p}=0.003)$ antibodies compared to patients without anti-KIR antibodies. Age at disease onset (21 vs $30, p=0.74)$, SLICC damage index $(1.5$ vs $1.0, p=0.31)$ or presence of anti-dsDNA $(70.0 \%$ vs $60.5 \%)$ were not significantly different.

Conclusion Autoantibodies to KIR-receptors are found in patients with SLE, pSS and SSc. Given the association with lupus nephritis such autoantibodies may have a clinical importance.

\section{PS1:17 LUPUS NEPHRITIS: SEVERELY REDUCED URINARY DNASE I LEVELS REFLECT LOSS OF RENAL DNASE I, DISEASE PROGRESSION AND MAY REDUCE THE NEED FOR RENAL BIOPSIES}

\footnotetext{
${ }^{1} \mathrm{HL}$ Pedersen, ${ }^{1} \mathrm{KD}$ Horvei, ${ }^{1} \mathrm{D}$ Thiyagarajan, ${ }^{2} \mathrm{GE}$ Norby, ${ }^{1} \mathrm{~N}$ Seredkina, ${ }^{3} \mathrm{G}$ Moroni, ${ }^{4} \mathrm{H}$ Holdaas, ${ }^{5} \mathrm{EH}$ Strøm, ${ }^{6} \mathrm{P}$-L Meroni, ${ }^{1} \mathrm{OP}$ Rekvig. ${ }^{1}$ Department of Medical Biology, UiT-the Arctic University of Norway, Tromso, Norway; ${ }^{2}$ Department of Rheumatology, OUS-the Oslo University Hospital, Oslo, Norway; ${ }^{3}$ Division of Nephrology and Dialysis, Ospedale Maggiore, Milan, Italy; ${ }^{4}$ Department of Medicine, Ous-the Oslo University Hospital, Oslo, Norway; ${ }^{5}$ Department of Pathology, OUS-the Oslo University Hospital, Oslo, Norway; ${ }^{6}$ Department of Clinical Sciences and Community Health, University of Milan and IRCCS Istituto Auxologico, Milan, Italy
}

10.1136/lupus-2018-abstract.65
Loss of renal DNase I leads to progression of lupus nephritis. Therefore, we determined if loss of renal DNase I reflects a concurrent loss of urinary DNase I, and whether absence of urinary DNase I predicts disease progression, which thus may reduce the need for renal biopsies. Here, mouse renal DNase I mRNA was determined by qPCR, whereas mouse and human DNase I protein and DNase I endonuclease activity levels were determined by Western blots, and gel and radial zymography assays, respectively, during different stages of the murine and human forms of the disease. Cellular localization of DNase I was analysed by immunohistochemistry, immunofluorescence, confocal microscopy and immune electron microscopy. We further compared DNase I levels in human native and transplanted kidneys to determine if the disease depended on autologous renal genes, or whether the nephritic process proceeded also in transplanted kidneys. We also analysed if DNase I levels in urine samples reflected expression levels in the kidneys, and if the mouse data were translatable to humans.

The data indicates that silencing of the renal DNase I gene expression level relates to serious progression of lupus nephritis in murine, human native, and transplanted kidneys. Notably, silencing of renal DNase I correlates with loss of DNase I protein and endonuclease activity in the urine samples. Thus, urinary DNase I levels reflects the renal DNase I expression and activity levels, and may therefore be used as a marker of lupus nephritis disease progression and reduce the need for renal biopsies.

\section{PS1:18 ECHOCARDIOGRAPHY FOR THE ASSESSMENT OF CARDIOVASCULAR BURDEN IN LUPUS: A SINGLE CENTRE COHORT STUDY}

${ }^{1} \mathrm{C}$ Ancuta, ${ }^{1,2} \mathrm{C}$ Pomirleanu, ${ }^{2} \mathrm{R}$ Paiu, ${ }^{3} \mathrm{E}$ Ancuta, ${ }^{1} \mathrm{C}$ lordache, ${ }^{4} \mathrm{R}$ Chirieac, ${ }^{1,5} \mathrm{~F}$ Mitu. ${ }^{1}$ University of Medicine and Pharmacy Grigore T Popa, lasi, Romania; ${ }^{2}$ Clinical Rehabilitation Hospital, Rheumatology Department, lasi, Romania; ${ }^{3}$ Elena Doamna Clinical Hospital, lasi, Romania; ${ }^{4}$ SANOCARE Medical and Research Centre, lasi, Romania; ${ }^{5}$ Clinical REhabilitatation Hospital, Cardiology Department, lasi, Romania

\subsection{6/lupus-2018-abstract.66}

Despite major achievements in understanding the pathobiology and management of Systemic Lupus Erythematosus (SLE), cardio-vascular burden remains a complex challenge in routine practice. Echocardiography emerges as a valuable non-invasive technique widely recommended for the screening, evaluation and monitoring of cardiac involvement in different SLE settings.

Objectives to evaluate the prevalence and nature of the clinical and subclinical cardiac involvement in SLE, and to identify potential relation with several disease-related parameters.

Design and methods Retrospective observational study in 120 consecutive SLE (fulfilling either 1987 ACR or new 2012 SLICC/ACR criteria), mean age $36.9+15.2$ years and mean disease duration $9.2+8.5$ years, attending the outpatient rheumatology department at least once. Demographic, clinical, immunologic profile, disease activity (SLEDAI), organ damage (SLICC/ACR) data were collected.

Patients were assessed according to a standardised protocol focused on clinical, immunologic profile, disease activity (SLEDAI), severity and organ damage (SLICC/ACR); cardiac involvement (valve damage, systolic and diastolic dysfunction, pericardial 
disease, myocardial ischemia, pulmonary hypertension) was evaluated by 2D transthoracic echocardiography (TTE).

Statistical analysis was done in SPSS, $\mathrm{p}<0.05$.

Results Valvular disease was reported in 61.98\% SLE (14\% stenosis, 20.66\% valvular masses, 33\% mild-to- moderate regurgitation, $34.73 \%$ leaflet thickening. Libman-Sacks endocarditis was not depicted. Asymptomatic decrease in left ventricular ejection fraction (55\%) was described in $16.52 \%$, the lowest LVEF being $33 \%$ in $4.13 \%$ patients; statistical significant negative correlation LVEF - disease duration and activity $(p<0.05)$ was found. LV diastolic dysfunction as subclinical cardiac involvement was registered in 59.50\% SLE, with a direct correlation with disease duration $(\mathrm{p}<0.05)$, but not with disease activity (SLEDAI) and organ damage (SLIC/ACR) $(p>0.05)$. Global hypokinesis on TTE as an indicator of subclincal myocarditis was demonstrated in up to one third SLE, while cardiomyopathy in one fourth. Mild pericardial effusion is observed in $31.2 \%$ cases, while cardiac tamponade in 4 cases; pericardial thickening was reported in 38\% SLE. Finally, abnormal systolic pressure in pulmonary artery was found in $24.79 \%$ patients.

Conclusion Patients with SLE are at increased risk to develop either clinical or subclinical cardiovascular manifestations as demonstrated by echocardiographic studies. A systematic TTE assessment is routinely recommended for the screening and monitoring of cardiac events.

\section{PS1:19 BIOLOGICAL DMARDS-INDUCED LUPUS IN PATIENTS WITH RHEUMATOID ARTHRITIS: A SINGLE CENTRE EXPERIENCE}

${ }^{1} \mathrm{C}$ Ancuta, ${ }^{1,2} \mathrm{C}$ Pomirleanu, ${ }^{2} \mathrm{R}$ Paiu, ${ }^{1} \mathrm{C}$ lordache, ${ }^{3} \mathrm{E}$ Ancuta, ${ }^{4} \mathrm{R}$ Chirieac. ${ }^{1}$ University of Medicine and Pharmacy Grigore T Popa, lasi, Romania; ${ }^{2}$ Clinical Rehabilitation Hospital, Rheumatology Department, lasi, Romania; ${ }^{3}$ Elena Doamna Clinical Hospital, lasi, Romania; ${ }^{4}$ Sanocare Medical and Research Centre, lasi, Romania

\subsection{6/lupus-2018-abstract.67}

Biological drugs (bDMARDs) have changed landscape and outcomes in various rheumatic conditions. Increased risk for infections, paradoxical reactions, autoimmunogenicity are widely acknowledged and may be responsible for treatment failure and switching among biologics.

Objective To assess drug-induced autoimmunity (serology, clinical significance) in patients with rheumatoid arthritis (RA) treated with bDMARDs.

Patients and methods Longitudinal observational study in 246 bionaïve RA receiving their first bDMARD, anti-tumour necrosis factor (TNF) or non-TNF drugs, according to local treatment guidelines.

Disease activity, therapeutic response and serial autoantibody profiles (antinuclear (ANA), anti-dsDNA) were systematically assessed at baseline and every six months. Prevalence, clinical significance and therapeutic implications of ANA/antidsDNA specificities were further analysed (SPSS-19, $\mathrm{p}<0.05$ ).

Results 214 RA received TNF inhibitors (56 infliximab, 65 adalimumab, 8 golimumab, 18 certolizumab, 67 etanercept) as first bDMADs and 32 different mechanism of action (10 rituximab, 7 abatacept, 15 tocilizumab). Overall, ANA seroconversion was demonstrated in $14.22 \%(35 / 246)$, while anti-dsDNA in $8.94 \%$ $(22 / 246)$; no change in the immunological profile under golimumab, certolizumab, abatacept or rituximab.
Significantly higher rates of ANA were reported in infliximabtreated RA (30.53\%) as compared to adalimumab (18.46\%), etanercept $(7.46 \%)$ and tocilizumab (6.66) $(\mathrm{p}<0.05)$; anti-dsDNA positivity was found in 10 infliximab (17.85\%), 4 adalimumab (12.30\%), 3 etanercept $(4.47 \%)$ and one tocilizumab (6.66\%), within an average of 36.5 (2-67) months.

ANA/anti-dsDNA positive status correlated with RA activity (DAS28-ESR) and loss of therapeutic response, concomitant DMARDs (leflunomide) and corticosteroids, administration adverse reactions $(\mathrm{p}<0.05)$.

Only four patients $(1.62 \% ; 4 / 246)$ were classified as druginduced lupus. Three of them (infliximab, adalimumab, tocilizumab -induced) developed mild to moderate disease, with characteristic malar rash, serositis, haematological, elevated ANA and anti-dsDNA that normalised six months after bDMARD discontinuation; the fourth patient had adalimumabinduced lupus two months after drug initiation, with severe course (subacute extensive skin lesions, cytopenia, serositis) and fatal outcome.

Conclusions Development of ANA with or without antidsDNA specificity is typically associated with loss of response to bDMARD in RA and infrequently responsible for druginduced lupus. Systematic evaluation of ANA/dsDNA is not recommended in routine practice, but essential in certain specific clinical settings (secondary non-responders or lupus-like symptoms).

\section{PS1:20 MINING FOR COMMON REACTIVITY PATTERNS OF HUMAN AUTOANTIBODIES AGAINST ENDOGENOUS PROTEIN TARGETS USING CLUSTERED AUTOANTIBODY REACTIVITIES}

H-D Zucht, P Budde, L Steeg, M Tuschen, S Bhandari, P Schulz-Knappe. Protagen AG, Dortmund, Germany

\subsection{6/lupus-2018-abstract.68}

Purpose Autoimmune diseases arise from an abnormal immune response of the body against self-proteins leading to tissue and organ damage. The excessive production of harmful autoantibodies ( $\mathrm{AAB})$ is a hallmark of autoimmune diseases including rheumatoid arthritis (RA), systemic lupus erythematosus (SLE), systemic sclerosis (SSc) and Sjogren's syndrome (SjS). Also in cancer research, it has been recently shown that AABs are useful to characterise patients. The characterisation of patient subgroups by means of stratification is essential for the efficient development of therapies, but often difficult due to the lack of appropriate biomarkers. Personalised or precision medicine approaches rely on appropriate multivariate multiplexing technology and data analysis. AABs serve as diagnostic markers for various autoimmune diseases, but the co-occurrence of $\mathrm{AABs}$ has rarely been analysed and is difficult to comprehend. Detecting a broad set of AABs helps to investigate the similarity between patients. A multiplex signature enables clustering for the investigation of relationships and patterns, which can be related to relevant clinical variables.

Methods Here, we illustrate Luminex bead-based AAB assays using a set of 96 biomarker targets and their utility to characterise SLE and SSc as well as cancer study groups.

Data analysis is based on bi-clustering algorithms and a prevalence and signature analysis of the markers. Cluster analysis was also performed using transformed data sets (qualitative) to 